\title{
Histomorphometric case-control study of subarticular osteophytes in patients with osteoarthritis of the hip
}

Rasmus Klose-Jensen ${ }^{1,2^{*}}$ (D), Andreas Wiggers Nielsen ${ }^{1}$, Louise Brøndt Hartlev ${ }^{1,3}$, Jesper Skovhus Thomsen ${ }^{4}$, Lene Warner Thorup Boel ${ }^{5}$, Mogens Laursen ${ }^{6}$, Kresten Krarup Keller ${ }^{1,7}$ and Ellen-Margrethe Hauge ${ }^{1,2}$

\begin{abstract}
Objective: The objective of this cross-sectional case-control study was to determine the prevalence and size of marginal and subarticular osteophytes in patients with osteoarthritis $(\mathrm{OA})$, and to compare these to that of a control group.

Design: We investigated femoral heads from 25 patients with OA following hip replacement surgery, and 25 femoral heads from a control group obtained post-mortem. The area and boundary length of the femoral head, marginal osteophytes, and subarticular osteophytes were determined with histomorphometry. Marginal osteophytes were defined histologically as bony projections at the peripheral margin of the femoral head, while subarticular osteophytes were defined as areas of bone that expanded from the normal curvature of the femoral head into the articular cartilage.

Results: The prevalence of OA patients with marginal- and subarticular osteophytes were 100 and 84\%, respectively. Whereas the prevalence of the participants in the control group with marginal- and subarticular osteophytes were 56 and $28 \%$, respectively.

The area and boundary length of marginal osteophytes was (median (Interquartile range)) $165.3 \mathrm{~mm}^{2}$ (121.4-254.0) $\mathrm{mm}^{2}$ and $75.1 \mathrm{~mm}(50.8-99.3) \mathrm{mm}$ for patients with OA compared to $0 \mathrm{~mm}^{2}(0-0.5) \mathrm{mm}^{2}$ and $0 \mathrm{~mm}(0-0.5) \mathrm{mm}$ for the control group $(P<0.001)$. For the subarticular osteophytes, the area and boundary length was $1.0 \mathrm{~mm}^{2}(0-4.4)$ $\mathrm{mm}^{2}$ and $1.4 \mathrm{~mm}(0-6.5) \mathrm{mm}$ for patients with OA compared to $0 \mathrm{~mm}^{2}(0-0.5) \mathrm{mm}^{2}$ and $0 \mathrm{~mm}(0-0.5) \mathrm{mm}$ for the control group $(P<0.001)$.

Conclusion: As expected, both marginal- and subarticular osteophytes at the femoral head, were more frequent and larger in patients with OA than in the control group. However, in the control group, subarticular osteophytes were more prevalent than expected from the minor osteophytic changes at the femoral head margin, which may suggest that subarticular osteophytes are an early degenerative phenomenon that ultimately might develop into clinical osteoarthritis.
\end{abstract}

Keywords: Osteoarthritis, Femoral head, Osteophytes

\footnotetext{
* Correspondence: raujen@rm.dk

${ }^{1}$ Department of Rheumatology, Aarhus University Hospital, Palle Juul-Jensens Boulevard 45, 8200 Aarhus, Denmark

2Department of Clinical Medicine, Aarhus University, Aarhus, Denmark

Full list of author information is available at the end of the article
}

(c) The Author(s). 2020 Open Access This article is licensed under a Creative Commons Attribution 4.0 International License, which permits use, sharing, adaptation, distribution and reproduction in any medium or format, as long as you give appropriate credit to the original author(s) and the source, provide a link to the Creative Commons licence, and indicate if changes were made. The images or other third party material in this article are included in the article's Creative Commons licence, unless indicated otherwise in a credit line to the material. If material is not included in the article's Creative Commons licence and your intended use is not permitted by statutory regulation or exceeds the permitted use, you will need to obtain permission directly from the copyright holder. To view a copy of this licence, visit http://creativecommons.org/licenses/by/4.0/ The Creative Commons Public Domain Dedication waiver (http://creativecommons.org/publicdomain/zero/1.0/) applies to the data made available in this article, unless otherwise stated in a credit line to the data. 


\section{Background}

Osteoarthritis (OA) is the most common joint disease, and $\mathrm{OA}$ of the hip afflicts $3-6 \%$ of the population over 50 years [1]. The disease is characterised by loss of articular cartilage, sclerosis of the subchondral bone and formation of osteophytes [2]. Importantly, it has been shown that subchondral bone sclerosis and formation of marginal osteophytes at the knee could be detected before the thickness of the articular cartilage changes and the joint space narrows [3, 4]. Marginal osteophyte formation is thought to occur by the proliferation of periosteal- and synovium-derived mesenchymal stem cells differentiating into chondrocytes that form cartilage and through endochondral ossification leads to bony projections [5]. However, the formation of marginal osteophytes is likely not the only example of bone formation in OA. It has been hypothesised, that microcracks in the subchondral bone plate and the calcified cartilage may reactivate the secondary ossification centre and lead to loss of cartilage through endochondral ossification [6]. Using quantitative backscattered electron imaging, Ferguson et al. found high density mineralised protrusions (HDMP) at the mineralising front of calcified cartilage in femoral heads from patients with OA [7]. These HDMP have also been found in the knee of patients with OA, using magnetic resonance imaging (MRI) and microcomputed tomography [8]. The HDMP emerged from the calcified cartilage and subchondral bone junction and could extend up to two-thirds of the articular cartilage thickness, resulting in cartilage degeneration [9]. The mechanism for this cartilage degeneration is currently unknown, but HDMP could perhaps be associated with endochondral ossification and subarticular osteophyte formation. McCauley et al. showed that subarticular osteophytes in the knee visualised with MRI were associated with articular cartilage defects [10]. Similarly to HDMP, these subarticular osteophytes were defined using MRI [11]. At present, it is unknown whether HDMP transforms into subarticular osteophytes or whether subarticular osteophytes actually are HDMP. Until now, subarticular osteophytes have not been investigated with quantitative histomorphometric methods.

We acknowledge that subarticular osteophytes exist and might be part of the early pathological changes in patients with osteoarthritis. To further elucidate this hypothesis, we quantified the boundary length and the area of the entire femoral head, the marginal osteophytes and the subarticular osteophytes in both patients with OA and a control group with similar sex and age distribution.

\section{Materials \& methods}

The article was designed in accordance with Strengthening the Reporting of Observational Studies in Epidemiology (STROBE) checklist [12].

\section{Study design and ethical considerations}

The study was a cross-sectional histomorphometry study of marginal- and subarticular osteophytes in the femoral heads obtained from 25 patients with OA and 25 deceased subjects with similar sex and age distribution.

The arthritic femoral heads were obtained from patients with primary hip OA, who underwent total hip replacement surgery at the Department of Orthopaedics, Farsoe Hospital, Denmark. Prior to surgery, clinical assessment of OA based on pain, stiffness, and physical function, Western Ontario and McMaster Universities Arthritis Index (WOMAC, VAS3.1) [13] scheme was fulfilled. The WOMAC questionnaire is based on 24 component item scores, and the scores were normalised to $100 \mathrm{~mm}$ using the following correction factors: the sum of pain score $\times 0.5$, the sum of stiffness score $\times 0.2$, and the sum of physical function score $\times 0.059$ [13]. The radiographic classification of $\mathrm{OA}$ in the hip joint was evaluated using the Kellgren-Lawrence grading scale, which grades OA between 0 to 4 depending on severity [14].

Macroscopically normal femoral heads from the control group were obtained at autopsy from individuals who had died suddenly from accidents or acute diseases at the Department of Forensic Medicine, Aarhus University, Denmark.

The Ethics Committee of Medical Research in Denmark Region (J. no. 10776) and The Danish Data Protection Agency (J.nr: 2003-41-3447) approved the study. Written and informed consents were obtained from the patients with OA before their hip replacement surgery.

\section{Participant inclusion and exclusion criteria Patients with osteoarthritis}

Inclusion criteria for the OA patients were the fulfilment of the American College of Rheumatology clinical and radiographic criteria for OA [15] and a referral to total hip replacement surgery. Exclusion criteria were known bone metabolic diseases, diabetes mellitus, malignant diseases, secondary OA or other joint diseases.

\section{Control group}

Inclusion criteria were newly deceased subjects with macroscopically healthy femoral heads. The participants from the control group were excluded if they had a history of high-energy pelvic trauma, known diagnosis of bone metabolic disease, diabetes mellitus, malignant diseases or other joint diseases were also excluded.

\section{Sample size}

This is the first study to quantify subarticular osteophytes by histomorphometry. Therefore, no data is available on relevant populations for which sample size 
calculations can be made. We used the same study population, which we have used previously for investigating bone turnover in relation to overlying cartilage deterioration [16].

\section{Outcome measures \\ Processing of tissue}

Immediately after the removal, the femoral heads were fixed in $70 \%$ ethanol and then processed according to stereological sampling of vertical uniform random sections [17], previously described in detail [16, 18-20]. In brief: the entire femoral heads were rotated around a vertical axis, which was perpendicular to the anatomical top of the femoral head. After choosing a random starting point, the femoral head was sawed using a diamond precisionparallel saw (Exakt Apparatebau, Norderstedt, Germany) into 7-mm-thick parallel slices, which were halved, and alternating left and right half slices were randomly selected for the following microscopic evaluation $[17,18]$. Depending on the size of the femoral head, a total of five to seven 7-mm-thick halved parallel slices were collected from both patients with OA and controls. Each of the five to seven 7-mm-thick halved parallel slices were embedded undecalcified in methylmethacrylate and cut into $7-\mu \mathrm{m}$-thick histological sections using a Jung model $\mathrm{K}$ microtome ( $\mathrm{R}$ Jung, Heidelberg, Germany) equipped with a tungsten microtome knife. The sections were mounted and stained with Masson-Goldner trichrome [18].

\section{Definition of tissue structures}

Marginal osteophytes were defined as bony projections at the peripheral margin of the femoral head (5) (Fig. 1). Subarticular osteophytes were defined as areas of bone, which expanded from the normal curvature of the femoral head into the cartilage, and which was not located in relation to the peripheral margin of the femoral head or the fovea capitis [21] (Fig. 1). The fovea capitis was excluded in the present study.

\section{Histomorphometry}

Data were collected using a light microscope (Nikon Eclipse 80i, Tokyo, Japan) equipped with a motorised specimen stage (Prior Proscan 11 TM, Rockland, MA, USA), a microcator (Heidenhain MT 1201, Traunreut, Germany), and a digital video camera (OlympusDP72, Tokyo, Japan) connected to a PC running the newCAST interactive stereology software (v. 3.4.1.0, Visiopharm, Hørsholm, Denmark). Sampling regions were automatically aligned in newCAST. Test points and test lines were superimposed on the digital images of the tissue sections and viewed on the PC monitor at a total magnification of $\times 121.64$. For each of the 7-mm-thick parallel tissue sections, the profile number of subarticular and marginal

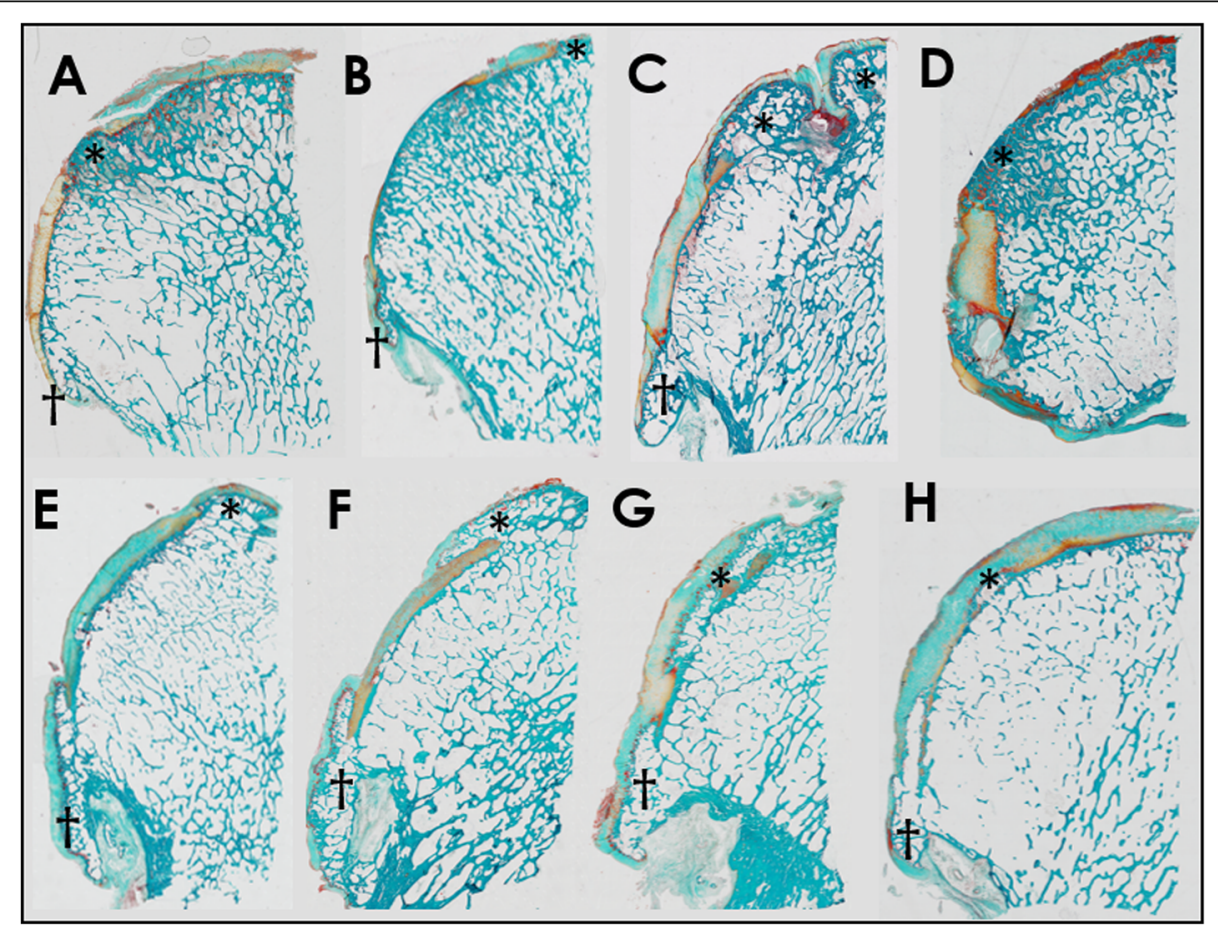

Fig. 1 Masson-Goldner trichrome stained 7- $\mu$ m-thick tissue section of a halved femoral heads. a 59-year-old male osteoarthritis (OA) patient. b 62-year-old female OA patient. c 56-year-old male OA patient. $\mathbf{d}$ 73-year-old female OA patient. e 67-year-old male OA patient. $\mathbf{f}$ and $\mathbf{g}$ 61-yearold male OA patient. $\mathbf{h} 66$-year-old female control. An asterisk denotes the subarticular osteophytes. A cross denotes the marginal osteophytes 
osteophytes were counted, and the area and boundary length of the femoral head, subarticular- and marginal osteophytes were estimated.

The total area $(A r)$ of the femoral head, subarticularand marginal osteophytes were estimated with point counting using all 7-mm-thick halved parallel tissue section from each individual femoral head. For estimations of the area of the subarticular and marginal osteophytes, a grid with an area per point of $0.49 \mathrm{~mm}^{2}$ was used. For estimations of the area of the femoral head, a grid with an area per point of $2.93 \mathrm{~mm}^{2}$ was used.

$$
A r=a(p) \times \sum_{i=1}^{n} p
$$

Where $n$ is the number of sections, $\sum_{i=1}^{n} p$ is the total number of test points hitting the structure of interest and $a(p)$ is the area per test point [22].

The total boundary length $(\mathrm{Bd})$ of the femoral head, subarticular- and marginal osteophytes from the five to seven 7-mm-thick halved parallel tissue section, were estimated using a line probe with an area per length of $0.297 \mathrm{~mm}$.

$$
B d=\pi / 2 \times \sum_{i=1}^{n} l \times a(l)
$$

Where $\pi / 2$ is a constant used for sine-weighted testlines, $n$ is the number of sections, $\sum_{i=1}^{n} l$ is the total number of intersections between the tissue surface and the sine-weighted test-line grid, and $a(l)$ is the area per test line length of the line-grid superimposed on the tissue section [22].

\section{Statistical methods}

Data were analysed using STATA 12 (StataCorp LP, College Station, TX, USA). Normal distribution of the data was investigated with Q-Q plots and histograms. Normally distributed data are presented as mean (95\% confidence interval), and differences between the two groups were tested for statistical significance using Student's $t$-test. Data, which was not normally distributed, are presented as median (Interquartile range (IQR)), differences between the two groups were tested for statistical significance using the Mann-Whitney $U$ test. Coefficient of variation $(\mathrm{CV})$ was calculated as the standard deviation of the two repeated measurements divided by the subject mean in order to investigate the intraobserver reliability of the measures. The results were considered statistically significant at $P<0.05$.

\section{Results}

Patients demographics are shown in Table 1.

The median profile number (Table 2), area and boundary length for both marginal- and subarticular osteophytes were significantly larger in the patients with OA than in the control group (Fig. 2). The prevalence of subjects with marginal- and subarticular osteophytes were significantly larger in the patients with OA than in the control group (Table 2). In addition, 19 of the 25 (79\%) patients with OA had more than one profile number of subarticular osteophytes, whereas in the control group only 7 of the $25(28 \%)$ participants had more than one profile number of subarticular osteophytes $(p=$ 0.001 ). The high median profile number and boundary length of subarticular osteophytes indicate an enlarged surface area of the femoral heads.

In the patients with OA, $83 \%$ of the subarticular osteophytes was superficially covered with articular cartilage. The remaining $17 \%$ had a denuded bone surface. In the control group, $96 \%$ of the subarticular osteophytes were superficially covered with articular cartilage. The remaining $4 \%$ had a denuded bone surface. The proportion of subarticular osteophytes with denuded bone did not differ between patients with $\mathrm{OA}$ and the control group $(p=0.082)$.

The prevalence of OA patients with marginal osteophytes was significantly higher than the prevalence of

Table 1 Participants demographics

\begin{tabular}{llll}
\hline & $\begin{array}{l}\text { Control } \\
(\boldsymbol{n}=\mathbf{2 5})\end{array}$ & $\begin{array}{l}\text { Osteoarthritis } \\
(\boldsymbol{n}=\mathbf{2 5})\end{array}$ & $\begin{array}{c}\boldsymbol{p} \text {-value } \\
\text { Female (\%) }\end{array}$ \\
Age (years, mean \pm SD) & 52 & 52 & 0.000 \\
$\quad$ Male & $61.5 \pm 7.8$ & $64.4 \pm 7.2$ & 0.175 \\
$\quad$ Female & $62.0 \pm 8.5$ & $64.3 \pm 6.7$ & 0.466 \\
Kellgren-Lawrence grade (mean \pm SD) & $61.0 \pm 7.4$ & $64.5 \pm 7.8$ & - \\
WOMAC Normalized NRS 3.0 (mm, mean \pm SD) & - & $3.8 \pm 0.4$ & - \\
$\quad$ Pain & - & $50 \pm 21$ & - \\
$\quad$ Stiffness & - & $56 \pm 29$ & - \\
Physical Function & - & $44 \pm 14$ & \\
\hline
\end{tabular}

BMI Body Mass Index, WOMAC The Western Ontario and McMaster Universities Arthritis Index 
Table 2 Marginal- and subarticular osteophytes

\begin{tabular}{|c|c|c|c|}
\hline & Control $(n=25)$ & Osteoarthritis $(n=25)$ & $P$-Value \\
\hline \multicolumn{4}{|l|}{ Marginal osteophytes } \\
\hline Prevalence of participants with marginal osteophytes, n (\%) & $7(28)$ & $25(100)$ & $<0.001$ \\
\hline Profile number of marginal osteophytes, number & 16 & 133 & $<0.001$ \\
\hline Marginal osteophytes, median profile number (IQR) & $0(0$ to 1$)$ & $5(5$ to 6$)$ & $<0.001$ \\
\hline \multicolumn{4}{|l|}{ Subarticular osteophytes } \\
\hline Prevalence of participants with subarticular osteophytes, n (\%) & $14(56 \%)$ & $21(84 \%)$ & $<0.001$ \\
\hline Profile number of subarticular osteophytes, number & 26 & 82 & 0.014 \\
\hline Subarticular osteophytes, median profile number (IQR) & 3 (2 to 4$)$ & $5(5$ to 6$)$ & $<0.001$ \\
\hline
\end{tabular}

IQR Interquartile range

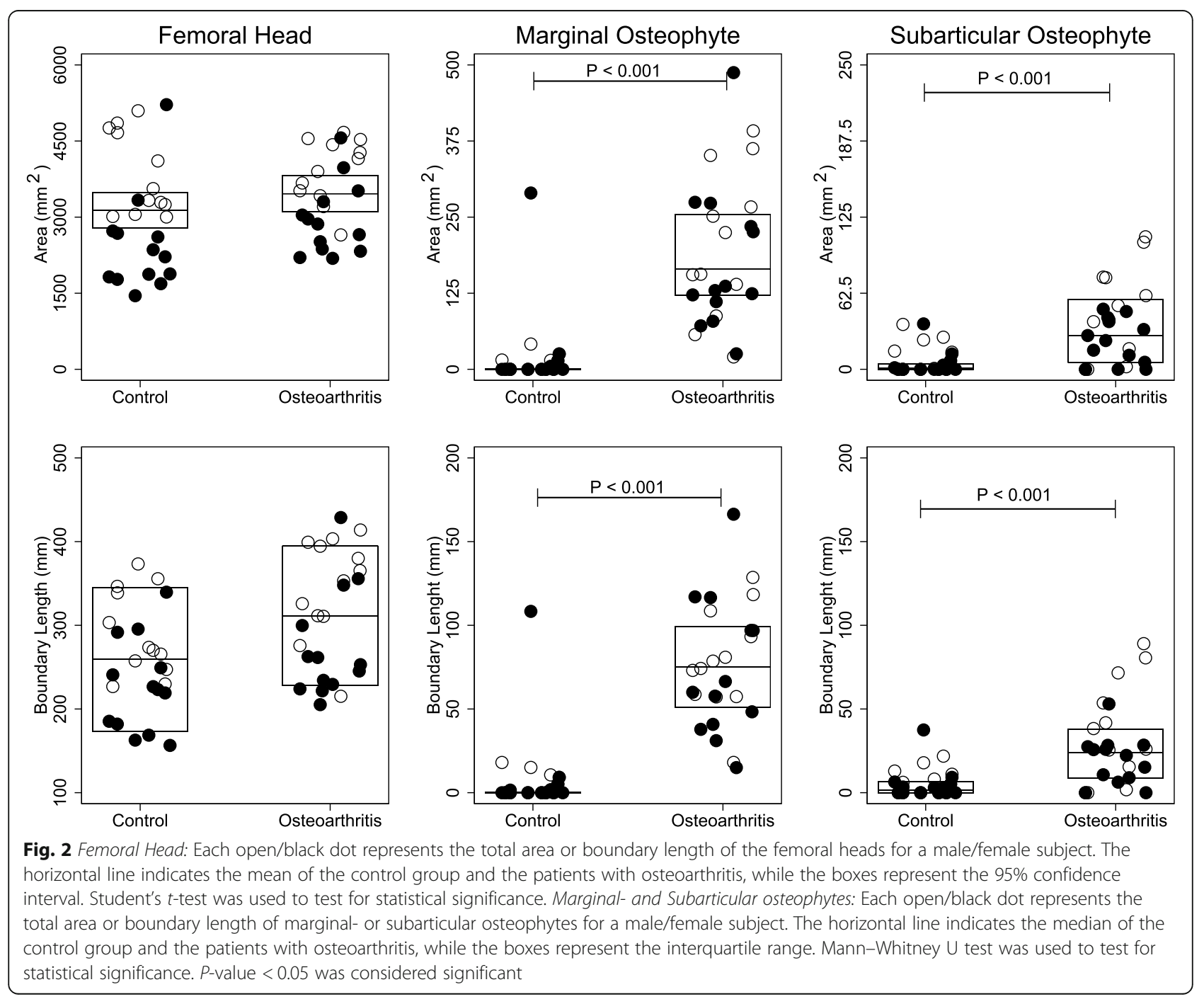


OA patients with subarticular osteophytes $(100 \%$ versus $84 \%, p=0.037$ ). Conversely in the control group, the prevalence of subjects with subarticular osteophytes was significantly higher than the prevalence of subjects with marginal osteophytes (56\% versus $28 \%, p=0.045$ ).

For the femoral head, marginal osteophytes and for the subarticular osteophytes the CV were 1.3, 9.4 and $28.4 \%$, respectively.

\section{Discussion}

In this cross-sectional study, we found that subarticular osteophytes protruding from the normal curvature of the femoral head into the articular cartilage were a common feature of patients with OA. Surprisingly, the control group with macroscopically healthy femoral heads also had subarticular osteophytes, but they were much less common and smaller than in patients with OA. Marginal osteophytes are a hallmark of osteoarthritis. This was clearly apparent in the present study as the patients with $\mathrm{OA}$ had larger and more frequent marginal osteophytes than the control group.

To our knowledge, subarticular osteophytes have not previously been quantified using histology. McCauley et al. investigated central osteophytes from 200 patients who were referred for MRI of the knee [10]. In that study, central osteophytes were defined as focal excrescences that extended from the cortical surface and were surrounded by articular cartilage on all sides. This definition is similar to the definition of subarticular osteophytes used in the present study. McCauley et al. found that subarticular osteophytes were associated with more articular cartilage defects and meniscal tears. Increased weight and age are known to predispose to osteoarthritis $[23,24]$, and the patients with subarticular osteophytes were also significantly older and had a higher Body Mass Index than patients without subarticular osteophytes [10]. The present study only investigated the femoral head. However, other studies have shown that central osteophytes also occur in the acetabulum, and the formation of central osteophytes occurred prior to joint space narrowing [25] according to Tönnis radiographic classification of hip OA [26].

In the present study, the subarticular osteophytes were slightly more common than marginal osteophytes in the control group. It has been shown that bone growth, in the form of marginal osteophytes, occurs before a loss of articular cartilage could be detected on conventional radiographs [3]. Therefore, it is not unreasonable to suggest that subarticular osteophytes also could be an early degenerative phenomenon which may occur before any loss of articular cartilage is evident, particularly as the size and frequency of subarticular osteophytes were greater in patients with $\mathrm{OA}$ than in the control group. Although the difference was not statistically significant,
$17 \%$ of the subarticular osteophytes had denuded bone in patients with OA compared to $4 \%$ of the control group indicating that the subarticular osteophytes form at the calcified cartilage invading the articular cartilage. Currently, the formation of marginal osteophytes, joint space narrowing, and subchondral bone sclerosis are the only features determined in radiographical OA assessment. However, these features may not be sufficiently sensitive to detect OA at an early stage, or sufficiently sensitive to change over time. Quantitative assessment of early osteoarthritic changes is necessary for investigating future medical therapies of OA. Therefore, we suggest that the subarticular osteophytes investigated in the present study might be an OA biomarker using quantitative computed tomography.

It is well known that marginal osteophytes grow by endochondral ossification with progressive changes of cellular proliferation, differentiation, and elaboration of intercellular matrix [21]. However, it is currently unknown how subarticular osteophytes form. A possible mechanism for the progression of subarticular osteophytes might be that they form as a consequence of microcracks in the calcified cartilage and subchondral bone [6], which might result in HDMP [11]. Supposing that HDMP is an early sign of the reactivation of the secondary ossification centre, the articular cartilage might be lost due to endochondral ossification as illustrated in Fig. 3, and not only as a consequence of fragmentation, wear and tear [7]. However, longitudinal studies are needed to investigate this hypothesis.

An important strength of the study is the use of systematic uniform random sampling. This sampling method ensures all positions in the complete human femoral heads are given equal probability of being sampled $[18,27]$. Thereby, we did not selectively study the surface based on its specific appearance as normal or degrees of abnormalities but instead based on its random sampling. Another strength of the study is the separate quantitative estimates of osteophyte area. Because we used 2D histomorphometry, we were not able to measure the unbiased volume and surface area of individual discrete osteophytes in the femoral heads [28, 29]. However, the profile number together with the unbiased total osteophytic area and boundary length still indicate the degree of osteophytic formation for the individual femoral heads. A weakness of the current study is its crosssectional design. Therefore, we can only speculate on how the subarticular osteophytes develop. Another weakness is that in areas devoid of articular cartilage, it was not possible to identify whether subarticular osteophytes were present or not. Thus, the estimates for the number of subarticular osteophytes in patients with OA were likely underestimated, while this was not the case for the control group. The control group was also 


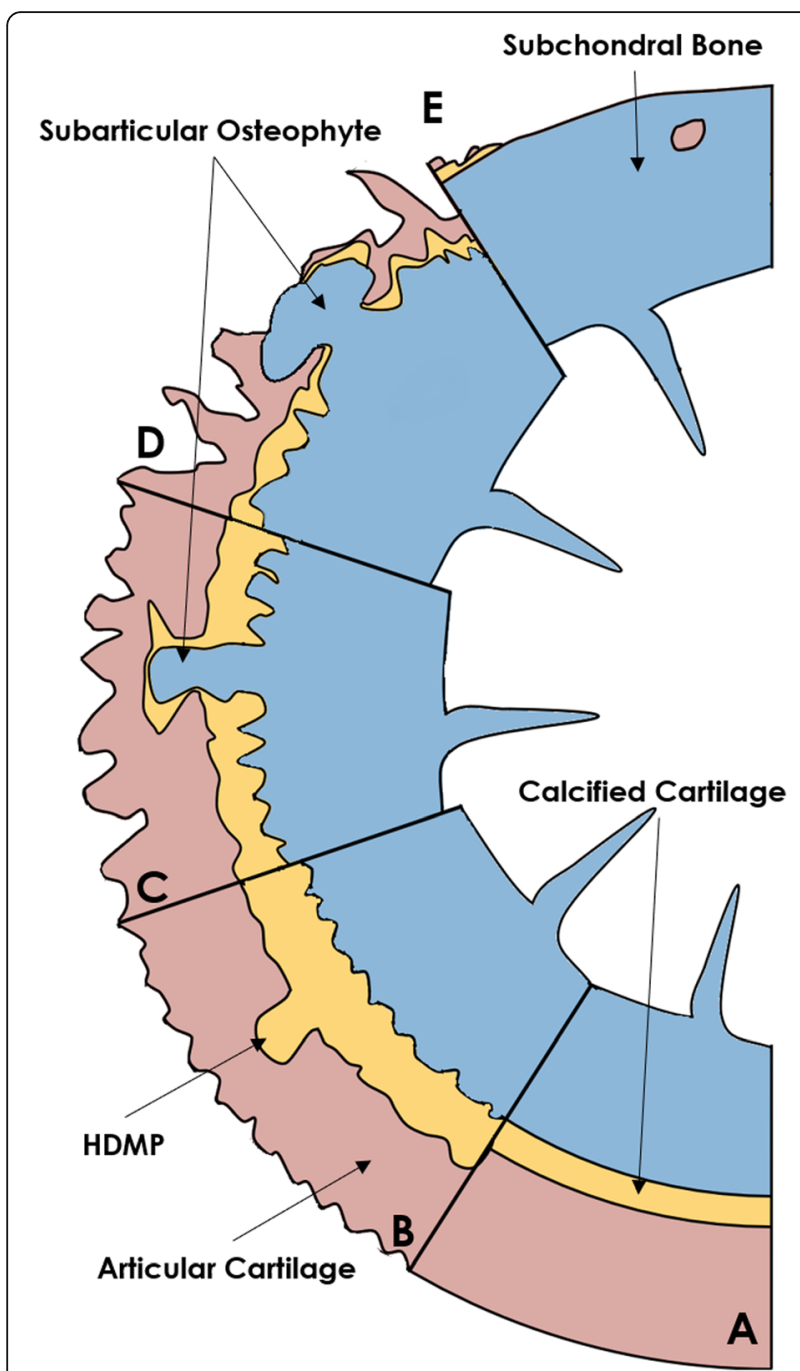

Fig. 3 Schematic illustration of the hypothesised growth of subarticular osteophytes into articular cartilage. a Healthy joint surface with articular cartilage, calcified cartilage and subchondral bone. $\mathbf{b}$ High density mineralised protrusions (HDMP) emerged from cracks in the calcified cartilage and subchondral bone. c Endochondral ossification of the high density mineralised protrusions resulting in subarticular osteophytes and superficial fibrillation of the articular cartilage. $\mathbf{d}$ The subchondral bone penetrates the articular cartilage. e Complete loss of the articular cartilage, and subchondral bone sclerosis

slightly younger than the OA patients, although this difference was not statistically significant. Lastly, although the control group had a macroscopically normal femoral head, it cannot be excluded that the control group might have preclinical OA leading to the presence of histologically identifiable osteophytic changes. Still, the changes in the control group could be a degenerative phenomenon associated with age as we have also shown histomorphometrically for minor cartilage deterioration [20].

\section{Conclusions}

In conclusion, the subarticular osteophytes were larger and more frequent in patients with osteoarthritis compared with the control group, suggesting that subarticular osteophytes are a common feature of OA pathology. Furthermore, in the control group, the frequency and size of subarticular osteophytes were more prevalent than expected, which may suggest that subarticular osteophytes might be an early degenerative phenomenon that might ultimately develop into clinical osteoarthritis. Consequently, subarticular osteophytes might possibly be used as an imaging marker for diagnosis and monitoring of early OA.

\section{Supplementary information}

Supplementary information accompanies this paper at https://doi.org/10. 1186/s12891-020-03648-w.

\section{Additional file 1}

\section{Abbreviations}

HDMP: High Density mineralised protrusions; MRI: Magnetic resonance imaging; OA: Osteoarthritis; OARSI: Osteoarthritis Research Society International; WOMAC: Western Ontario, and McMaster Universities Arthritis Index

\section{Acknowledgements}

The authors are grateful for the technical assistance of Jette Barlach and Rita Ullerup.

\section{Authors' contributions}

All authors were involved in drafting the article or revising it critically for important intellectual content, and all authors approved the final version to be published. Study conception and design: RKJ, KKK, and EMH. Acquisition of Tissue: LWTB, MB, LBH, and EMH. Analysis and interpretation of data: RKJ, KKK, EMH, AWN and JST.

\section{Funding}

The study was produced without funding.

Availability of data and materials

The datasets generated during and/or analysed during the current study are available from the corresponding author on reasonable request.

\section{Ethics approval and consent to participate}

The Ethics Committee of Medical Research in Denmark Region (J. no. 10776) and The Danish Data Protection Agency (J.nr: 2003-41-3447) approved the study. Written and informed consents were obtained from the patients with OA before their hip replacement surgery.

\section{Consent for publication}

Not applicable.

\section{Competing interests}

Ellen-Margrethe Hauge reports personal fees from MSD, personal fees from Pfizer, personal fees from UCB, personal fees from Sobi, grants from Roche, grants from Novartis, outside the submitted work. Kresten Krarup Keller reports speaking fee from Pfizer. Rasmus Klose Jensen, Andreas Wiggers Nielsen, Louise Brøndt Hartlev, Lene Warner Thorup Boel, Mogens Berg Laursen and Jesper Skovhus Thomsen have no conflict of interest to declare.

\section{Author details}

${ }^{1}$ Department of Rheumatology, Aarhus University Hospital, Palle Juul-Jensens Boulevard 45, 8200 Aarhus, Denmark. ${ }^{2}$ Department of Clinical Medicine, Aarhus University, Aarhus, Denmark. ${ }^{3}$ Department of Clinical Medicine, 
Randers Regional Hospital, Randers, Denmark. ${ }^{4}$ Department of Biomedicine Anatomy, Aarhus University, Aarhus, Denmark. ${ }^{5}$ Institute of Forensic Medicine, Aarhus University, Aarhus, Denmark. ${ }^{6}$ Orthopaedic Surgery Research Unit, Aalborg University Hospital, Aalborg, Denmark. ${ }^{7}$ Diagnostic Centre, Silkeborg Regional Hospital, Silkeborg, Denmark.

Received: 20 April 2020 Accepted: 15 September 2020

Published online: 06 October 2020

\section{References}

1. Kim C, Linsenmeyer KD, Vlad SC, Guermazi A, Clancy MM, Niu J, et al. Prevalence of radiographic and symptomatic hip osteoarthritis in an urban United States community: the Framingham osteoarthritis study. Arthritis Rheumatol. 2014;66:3013-7.

2. Martel-Pelletier J, Barr AJ, Cicuttini FM, Conaghan PG, Cooper C, Goldring MB, et al. Osteoarthritis. Nature Rev Disease Primers. 2016;2:1-18. https://doi. org/10.1038/nrdp.2016.72.

3. Buckland-Wright JC, Lynch JA, Dave B. Early radiographic features in patients with anterior cruciate ligament rupture. Ann Rheum Dis. 2000;59(8): 641-6.

4. Buckland-Wright C. Subchondral bone changes in hand and knee osteoarthritis detected by radiography. Osteoarthr Cartil. 2004;12(Suppl A): S10-9.

5. van der Kraan PM, van den Berg WB. Osteophytes: relevance and biology. Osteoarthr Cartil. 2007;15:237-44.

6. Burr DB, Radin EL. Microfractures and microcracks in subchondral bone: are they relevant to osteoarthrosis? Rheum Dis Clin N Am. 2003;29:675-85.

7. Ferguson VL, Bushby AJ, Boyde A. Nanonmechanical properties and mineral concentration in articular calcified cartilage and subchondral bone. J Anat. 2003:203:191-202.

8. Thomas NP, Jeffery N, van't Hof R, Adams VL, Saers J, Ranganath LR, et al. An Investigation of High Density Mineralised Protrusions in Human Cadaveric Knees by MRI and Microtomography. Osteoarthr Cartil. 2017;25: S253-4. https://doi.org/10.1016/j.joca.2017.02.427.

9. Boyde A, Riggs CM, Bushby AJ, McDermott B, Pinchbeck GL, Clegg PD. Cartilage damage involving extrusion of mineralisable matrix from the articular calcified cartilage and subchondral bone. Eur Cells Mater. 2011;21: 470-8.

10. McCauley TR, Kornaat PR, Jee WH. Central osteophytes in the knee: prevalence and association with cartilage defects on MR imaging. AJR Am J Roentgenol. 2001;176:359-64.

11. Boyde A, Davis GR, Mills D, Zikmund T, Cox TM, Adams VL, et al. On fragmenting, densely mineralised acellular protrusions into articular cartilage and their possible role in osteoarthritis. J Anat. 2014:225:436-46.

12. Sharp SJ, Poulaliou M, Thompson SG, White IR, Wood AM. A review of published analyses of case-cohort studies and recommendations for future reporting. PLoS One. 2014;9:e101176. https://doi.org/10.1371/journal.pone. 0101176.

13. Bellamy N, Buchanan WW. A preliminary evaluation of the dimensionality and clinical importance of pain and disability in osteoarthritis of the hip and knee. Clin Rheumatol. 1986;5:231-41.

14. Kellgren JH, Lawrence JS. Radiological assessment of osteo-arthrosis. Ann Rheum Dis. 1957;16:494-502. https://doi.org/10.1136/ard.16.4.494.

15. Altman R, Alarcón G, Appelrouth D, Bloch D, Borenstein D, Brandt K, et al. The American College of Rheumatology criteria for the classification and reporting of osteoarthritis of the hip. Arthritis Rheum. 1991;34:505-14.

16. Klose-Jensen R, Hartlev LB, Boel LWT, Laursen MB, Stengaard-Pedersen K, Keller KK, et al. Subchondral bone turnover, but not bone volume, is increased in early stage osteoarthritic lesions in the human hip joint. Osteoarthr Cartil. 2015;23:2167-73.

17. Baddeley AJ, Gundersen HJG, Cruz-Orive LM. Estimation of surface area from vertical sections. J Microsc. 1986;142:259-76.

18. Hartlev LB, Nyengaard JR, Thomsen JS, Boel LWT, Stengaard-Pedersen K, Hauge E-M. Application of design-based stereology for estimation of absolute volume and surface area of the articular and calcified cartilage compartments of undecalcified human femoral heads. J Microsc. 2013;251: 133-43.

19. Hartlev LB, Klose-Jensen R, Thomsen JS, Nyengaard JR, Boel LWT, Laursen $M B$, et al. Thickness of the bone-cartilage unit in relation to osteoarthritis severity in the human hip joint. RMD open. 2018;4:e000747. https://doi.org/ 10.1136/rmdopen-2018-000747.
20. Nielsen AW, Klose-Jensen R, Hartlev LB, Boel LWT, Thomsen JS, Keller KK, et al. Age-related histological changes in calcified cartilage and subchondra bone in femoral heads from healthy humans. Bone. 2019;129:115037. https://doi.org/10.1016/j.bone.2019.115037.

21. Jeffery AK. Osteophytes and the osteoarthritic femoral head. J Bone Joint Surg Br. 1975;57-B:314-24. https://doi.org/10.1302/0301-620X.57B3.314.

22. Howard CV, Reed MG. Unbiased Stereology. Three-Dimensional Measurements in Microscopy. 2nd edition. Oxford: Garland Science/Bios Scientific Publishers; 2005.

23. Reyes C, Leyland KM, Peat G, Cooper C, Arden NK, Prieto-Alhambra D. Association between overweight and obesity and risk of clinically diagnosed knee, hip, and hand osteoarthritis: a population-based cohort study. Arthritis Rheumatol. 2016;68(8):1869-75.

24. Neogi T, Zhang Y. Epidemiology of osteoarthritis. Rheum Dis Clin N Am. 2013;39:1-19. https://doi.org/10.1016/j.rdc.2012.10.004

25. Mofidi A, Shields JS, Stubbs AJ. Central acetabular osteophyte (saber tooth sign), one of the earliest signs of osteoarthritis of the hip joint. Eur J Orthop Surg Traumatol. 2011;21:71-4.

26. Kovalenko B, Bremjit P, Fernando N. Classifications in brief: Tonnis classification of hip osteoarthritis. Clin Orthop Relat Res. 2018;476:1680-4. https://doi.org/10.1097/01.blo.0000534679.75870.5f.

27. Gundersen HJ, Jensen EB. The efficiency of systematic sampling in stereology and its prediction. J Microsc. 1987;147(Pt 3):229-63.

28. Sterio DC. The unbiased estimation of number and sizes of arbitrary particles using the disector. J Microsc. 1984;134(Pt 2):127-36.

29. Hauge EM, Mosekilde L, Melsen F. Stereological considerations concerning the measurement of individual osteoid seams and resorption cavities. Bone Miner. 1994;27(3):249.

\section{Publisher's Note}

Springer Nature remains neutral with regard to jurisdictional claims in published maps and institutional affiliations.

Ready to submit your research? Choose BMC and benefit from:

- fast, convenient online submission

- thorough peer review by experienced researchers in your field

- rapid publication on acceptance

- support for research data, including large and complex data types

- gold Open Access which fosters wider collaboration and increased citations

- maximum visibility for your research: over $100 \mathrm{M}$ website views per year

At $\mathrm{BMC}$, research is always in progress.

Learn more biomedcentral.com/submissions 